\title{
Clinical Trial Highlights: Targeting Alpha-Synuclein
}

Kevin McFarthing

Parkinson's Advocate, Innovation Fixer Ltd, Oxford, UK

kevin(dot)mcfarthing(at)googlemail(dot)com

Tanya Simuni

Parkinson's Disease and Movement Disorders Center,

Northwestern University Feinberg School of Medicine, Chicago, USA

\section{Introduction}

Accumulation of alpha-synuclein ( $\alpha$-synuclein) in cells is the pathological hallmark of Parkinson's Disease (PD). There is mounting evidence that $\alpha$-synuclein is not simply a marker but it plays a key role in the pathogenesis of the disease. Aggregated $\alpha$-synuclein can interfere with the normal autophagy and lysosomal degradation functions of the cells, thus contributing to cell loss and can also spread intercellularly. As such, $\alpha$-synuclein has become the major target of therapeutic developments in PD. $\alpha$-synuclein targeted therapeutics can be divided into broad categories of

1. Reduction of extracellular $\alpha$-synuclein.
a. Passive immunization.
b. Active immunization.

2. Blocking misfolding of $\alpha$-synuclein (NPT200-11).

3. Blocking $\alpha$-synuclein aggregation (Nilotinib).

4. Reduction of $\alpha$-synuclein synthesis (siRNAs and ASO) - not in clinical phase yet.

Immunotherapies for Parkinson's are currently in the early stages of clinical development (Phases 1 and 2). Four of the five projects (Astra Zeneca, Biogen, Lundbeck and Roche) are based on passive therapy, i.e. the administration of already prepared antibodies. The only active immunization approach currently in clinic is from Affiris, using synthetic peptides to mimic epitopes on $\alpha$-synuclein. Affiris' PD01A has successfully completed Phase 1, displaying good levels of immunogenicity. No efficacy data were generated.

The most advanced projects are BIIB054 from Biogen (the SPARK study) and Prasinezumab from Roche (the PASADENA study). Both are in phase 2 and are recruiting 300 or so participants, but with different study objectives. The PASADENA trial aims to assess early efficacy as measured by the change in MDSUPDRS as the primary outcome, whereas SPARK's primary objective is assessment of safety and tolerability. Both SPARK and PASADENA are collecting a rich data set of clinical, imaging, biomarkers and wearable technology data to inform the design of future studies. They are recruiting early stage Parkinson's patients yet to start dopaminergic therapy and not likely to need it for the duration of the study.

The other clinical phase projects investigating passive immunotherapy from Astra Zeneca and Lundbeck are in Phase 1, and accordingly outcomes are focused on safety.

The presence of only one project investigating $\alpha$-synuclein clearance via active immunotherapy in clinical phase may place this approach at a disadvantage compared to passive antibody therapy. The latter is also more likely to require more frequent administration in clinical practice. United Neuroscience's endobody technology is also an active immunization approach; their UB312 project is planned to enter a phase 1 trial in 2019. BAN0805, a passive antibody targeting $\alpha$-synuclein fibrils, is under development by BioArctic and Abbvie and is also slated to potentially enter to phase 1 in 2019. 
Many questions remain unanswered. We do not yet know whether immunogenicity will prompt clearance of $\alpha$-synuclein, or if the presence of passive antibodies in CSF together with a decrease in circulating $\alpha$-synuclein will translate to an influence on the progression of Parkinson's. We will need to understand the impact on both motor and non-motor symptoms.

There are other ways to target $\alpha$-synuclein toxicity being tested in early phase clinical trials. NPT200-11 is a small molecule inhibitor of $\alpha$-synuclein misfolding. A phase 1 study reported in 2016 that it is well tolerated at levels above those that produced robust pre-clinical results. Neuropore are collaborating with UCB on the development of NPT200-11 and a phase 1b study is the next milestone.

Nilotinib, currently used in the treatment of Chronic Myeloid Leukemia (CML), facilitates clearance of misfolded $\alpha$-synuclein by enhancing autophagy through c-Abl phosphorylation and parkin activation. A small uncontrolled phase 1 study in a group of patients with advanced Parkinson's disease and dementia and Lewy body disease produced interesting results, sufficient to prompt the initiation of two randomized studies both still focused on safety as the primary outcomes.

Sun Pharma is developing a novel c-Abl inhibitor currently in Phase I trial and slated to launch Phase 2 study shortly. Enterin's ENT-01 is currently in phase 2a as a symptomatic therapy targeting the relief of constipation in Parkinson's (the RASMET study) but the mechanism of action is that ENT-01 acts locally on $\alpha$-synuclein in enteric nerves.

Long term safety will be a key question. $\alpha$-synuclein is, after all, an abundant protein performing essential tasks, until it starts to misbehave in Parkinson's. Despite these unanswered questions, therapies targeting $\alpha$-synuclein are amongst the most promising and exciting projects currently under way.

The summaries below provide information on the on-going clinical trials as of the date of this review (January 2019). Information was largely derived from clinicaltrials.gov. and company websites unless stated otherwise. The summaries are presented first for immunotherapies and then for other mechanistic targets in alphabetical order of the manufacturing company for each category. 


\section{IMMUNOTHERAPIES}

\section{AFFIRIS - PD01A and PD03A}

BACKGROUND: Affiris are developing AFFITOPE® PD01A and PD03A, synthetic peptides that mimic epitopes on $\alpha$-synuclein, producing an active immune response. Both have been evaluated in Phase 1 studies in both early Parkinson's participants and a separate group with Multiple System Atrophy (MSA).

\section{PD01A}

TITLE: Phase 1 study series of PD01 in early Parkinson's Disease.

STATUS: Complete.

\section{CLINICALTRIALS.GOV ID FOR EACH STUDY:}

NCT01568099 - Tolerability and Safety of Sub-Cutaneous Administration of Two Doses of AFFITOPE® PD01A in Early Parkinson's Disease (AFF008).

NCT01885494 - Observational Phase 1b Follow-up Extension Study for Patients with Parkinson's Disease After Immunization with AFFITOPE® PD01A (AFF008E).

NCT02216188 - Follow-up Study to Assess One Boost Immunization with AFFITOPE® PD01A with Regard to Safety and Clinical Activity (AFF008A).

NCT02618941 - Follow-up Study to Assess a Second Boost Immunization with AFFITOPE® PD01A with Regard to Safety and Clinical Activity (AFF008AA).

SPONSOR: Affiris.

ENROLMENT: 32 patients.

STUDY DESIGN: The Phase 1 AFF008 series comprised 4 consecutive studies - AFF008, AFF008E, AFF008A and AFF008AA. In total, 32 patients were enrolled, 24 of them receiving active treatment and 8 PD patients only on standard of care medication, who served as an observational comparison group.

The active group were randomized to receive four immunizations with either low dose $(15 \mu \mathrm{g})$ or high dose $(75 \mu \mathrm{g})$ AFFITOPE® PD01A in intervals of four weeks on an outpatient basis (study AFF008). Eight patients were in an untreated, observational control group.

Twenty-one patients in the PD01A treatment groups and five in the observational group completed the entire series of studies. At screening, the average time of PD after the first diagnosis was 2.6 years. Patients were allowed to continue their standard of care PD medication.

An additional observational follow-up period of twelve months was added (study AFF008E). Part three of the study series (AFF008A) administered a booster immunization after rerandomizing patients from study AFF008E

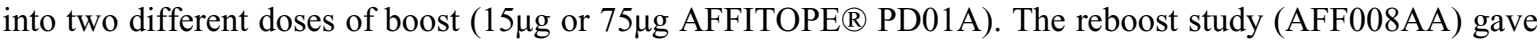
a second booster with a fixed dose of $75 \mu \mathrm{g}$ AFFITOPE® PD01A to patients previously immunized five times. Key objectives were to show safety and tolerability as well as immunogenicity of AFFITOPE ${ }^{\circ}$ PD01A in a long-term treatment setting.

OUTCOME: Both doses of AFFITOPE® PD01A were locally and systemically well tolerated ${ }^{1}$. No study drugrelated serious adverse events (SAE) or suspected unexpected serious adverse reactions (SUSAR) occurred. Systemic adverse events were very similar to those in patients on standard of care treatment. The majority of adverse events, approximately 55\%, were local reactions (LRs), the great majority of LRs being only mild and without dose dependency.

AFFITOPE ${ }^{\circledR}$ PD01A showed a clear immune response against the peptide itself and cross-reactivity against $\alpha$-synuclein targeted epitope over time. The first boost immunization produced a significant effect on all analysed 
titers, resulting in the maximum titers observed in this series of studies. The second boost immunization further stabilized the produced antibody titers. Thus, a significant increase in titers against PD01A was seen over time, which translated into a humoral immune response against $\alpha$-synuclein, being approximately one order of magnitude lower. In addition, PD01-specific antibodies were detectable in cerebrospinal fluid.

Data from post-hoc analyses indicate that AFFITOPE® PD01-induced antibodies preferentially bind to both oligomeric and fibrillar $\alpha$-synuclein when compared with monomers and showed that there was a trend in reduction of oligomeric $\alpha$-synuclein levels in plasma as well as cerebrospinal fluid upon treatment with PD01A at week 26.

Clinical scores for PD were stable during the entire study period, however, the study was not designed and not powered to evaluate clinical efficacy.

The logical next step is a Phase 2 study to further evaluate safety and tolerability and to gain early insight into clinical efficacy.

\section{PD03A}

TITLE: Study Assessing Tolerability and Safety of AFFITOPE® PD03A in Patients with Early Parkinson's Disease (AFF011).

OBJECTIVE: To assess safety and tolerability in Patients with Early Parkinson's Disease (AFF011).

STATUS: Complete.

CLINICALTRIALS.GOV ID: NCT02267434.

SPONSOR: Affiris.

ENROLMENT: 36 patients.

STUDY DESIGN: This study was a randomized, placebo-controlled, parallel group, patient-blinded, two centre study, assessing tolerability and safety of repeated subcutaneous (s.c.) administration of two doses of AFFITOPE® PD03A formulated with adjuvant to patients with early Parkinson's disease (PD).

Thirty-six patients were randomized to either AFFITOPE® PD03A high dose $(75 \mu \mathrm{g})$, low dose $(15 \mu \mathrm{g})$ or to the placebo group treated with adjuvant alone. Patients received 5 injections, 4 for priming every 4 weeks and the 5th as boost immunization 9 months after the first immunization. Key objectives were to show safety and tolerability as well as immunogenicity of AFFITOPE® PD03A.

At screening, the average time of PD after first diagnosis was between 1.6-2.3 years; patients were allowed to continue their standard of care PD medication.

OUTCOME: Both doses were locally and systemically well tolerated. No study drug related SAE or SUSAR occurred $^{2}$.

AFFITOPE $®$ PD03A showed a clear dose dependent immune response against the peptide itself and crossreactivity against the aSyn targeted epitope over time, with antibody reactivation upon booster immunization.

A parallel Phase 1 study (NCT02270489; AFF009) evaluated high dose (75 $\mu \mathrm{g})$ AFFITOPE® PD01A and PD03A in patients with MSA. Both doses were safe and well-tolerated. PD01A showed a clear immune response but, in contrast to the results in the PD trial, PD03A showed no difference to placebo ${ }^{3}$.

\section{COMMENTS:}

Affiris is taking a different approach of active vaccination to induce an antibody response, rather than passive antIbody delivery. There are potential advantages and disadvantages of such an approach. On the positive side the antibodies will bind specifically to a relevant appropriate target and they can be calibrated for specificity and 
selectivity of the immune response. In addition the drug is delived subcutaneously rather than intravenously and boosts can be done to further induce immune response. The potential pitfalls are lack of accurate prespecified level of immune response in a given individual and as such potential safety concerns though none have been seen so far in the early programs.

The Michael J Fox Foundation has reported that Affiris is planning a Phase 2 study $^{4}$.

\section{References}

1. http://www.affiris.com/news/affiris-announces-encouraging-long-term-data-from-a-series-of-first-inhuman-studies-using-affitope-pd01a-targeting-oligomeric-alpha-synuclein-in-early-parkinsons-diseasepatients/.

2. http://www.affiris.com/news/affiris-announces-top-line-results-of-first-in-human-clinical-study-usingaffitope/.

3. http://www.affiris.com/news/affiris-announces-results-of-a-phase-i-clinical-study-using-affitopes-pd01aand-pd03a/.

4. https://www.michaeljfox.org/foundation/news-detail.php?latest-trials-against-top-parkinson-proteinalpha-synuclein.

5. Schneeberger A, Tierney L, Mandler M Active immunization therapies for Parkinson's disease and multiple system atrophy. Mov Disord. 2016 Feb;31(2):214-24.

\section{ASTRA ZENECA - MEDI1341}

TITLE: A Randomized, Double-blind, Placebo-controlled Study of the Safety, Tolerability, Pharmacokinetics, and Pharmacodynamics of Single Ascending Doses of MEDI1341 in Healthy Male and Female Volunteers. Phase I.

STATUS: Recruiting.

CLINICALTRIALS.GOV ID: NCT03272165.

SPONSOR: AstraZeneca.

ENROLMENT: 40-48.

ESTIMATED COMPLETION DATE: November 2019.

OBJECTIVE: To evaluate safety and tolerability, pharmacokinetics, and pharmacodynamics of Single Ascending Doses (SAD) of MEDI1341 in Healthy Volunteers.

BACKGROUND: MEDI1341 is an $\alpha$-synuclein antibody.

STUDY DESIGN: This is a single-center study of single ascending intravenous doses of MEDI1341 or placebo in up to 48 healthy volunteers, aged 18 to 80 years. The study will include up to 5 planned cohorts; each cohort will comprise 8 subjects. An optional sixth cohort of 8 subjects may be studied. Each subject will receive a single 60-minute intravenous infusion of MEDI1341 or placebo and will undergo scheduled assessments over a period of 13 weeks. The main aim of the study is to assess the safety and tolerability of single doses of MEDI1341 in Healthy Volunteers.

\section{OUTCOME MEASURES:}

PRIMARY OUTCOME MEASURE: Adverse events.

SECONDARY OUTCOME MEASURES: spectrum of pharmacokinetic and pharmacodynamics measures including free/total $\alpha$-synuclein concentration in cerebrospinal fluid and plasma. Antidrug antibodies in serum will be measured as well. 


\section{COMMENTS:}

This is an early phase Single Ascending Dose (SAD) study that will define viability of the molecule to move into the more advanced stages of the development. MEDI1341 is an $\alpha$-synuclein antibody that is potentially differentiated by its high affinity, high selectivity and reduced effector function (lower interaction with the immune system). The impact of this differential profile will be determined in future studies.

\section{REFERENCES: No publications.}

\section{BIOGEN - BIIB054}

TITLE: Evaluating the Safety, Pharmacokinetics, and Pharmacodynamics of BIIB054 in Participants with Parkinson's Disease (SPARK) - Phase 2.

STATUS: Recruiting. More details on participation can be found at www.thesparkstudy.com/en-us/screener. html.

CLINICALTRIALS.GOV ID: NCT03318523.

SPONSOR: Biogen.

ENROLMENT: 311 patients.

ESTIMATED COMPLETION DATE: April 2022.

OBJECTIVE: To evaluate the dose-related safety of BIIB054; to evaluate the pharmacodynamic effects of BIIB054 on the integrity of nigrostriatal dopaminergic nerve terminals; to assess the pharmacokinetic (PK) profile of BIIB054; and to evaluate the immunogenicity of BIIB054.

BACKGROUND: BIIB054 is a human monoclonal antibody that targets aggregated forms of $\alpha$-synuclein. Pre-clinical work ${ }^{1}$ showed $>800$-fold binding to aggregated $\alpha$-synuclein compared to monomer, with epitope mapping showing that the antibody binds to residues 1-10. BIIB054 treatment in three different mouse models showed that it reduces the spread of $\alpha$-synuclein pathology, improves motor function and reduces the loss of dopamine transporter density in dopaminergic terminals.

The successful pre-clinical work supported the initiation of a Phase 1 trial (NCT02459886). This study, entitled "Single Ascending Dose Study of BIIB054 in Healthy Participants and Early Parkinson's Disease", was randomized, double-blind and placebo-controlled. Eighteen patients received a single dose of $15 \mathrm{or} 45 \mathrm{mg} / \mathrm{kg}$ of BIIB054, or placebo. Results ${ }^{2}$ showed favourable pharmacokinetic, safety and tolerability, with a serum halflife of 30 days.

STUDY DESIGN: SPARK is a multi-centre, randomized, double-blind, placebo-controlled study, with an active treatment dose-blinded period, to evaluate the safety, pharmacokinetics and pharmacodynamics of BIIB054 in subjects with Parkinson's.

The study is conducted in patients who have been diagnosed with clinically established PD within a maximum of 3 years with no other known or suspected cause of Parkinsonism. Patients should have a score of $\leq 2.5$ on the Modified Hoehn and Yahr Scale.

There will be 2 cohorts. Cohort A will be randomized first and will include approximately 24 patients (completed). Cohort B (on-going) was launched after all patients in Cohort A completed Week 12 assessments and had safety read out. Cohort 2 will include approximately 287 patients.

In Year 1 (the placebo-controlled portion of the study), patients will be randomized into the following 4 parallel dosing arms, to receive treatment every 4 weeks via IV infusion:

Arm 1: Placebo.

Arm 2: BIIB054 - 250 mg. 
Arm 3: BIIB054 - $1250 \mathrm{mg}$.

Arm 4: BIIB054 - $3500 \mathrm{mg}$.

Patients will continue treatment in Year 2 (the active-treatment dose-blinded portion of the study). Those who received placebo in Year 1 will be randomized to one of the active-treatment arms to receive BIIB054 in Year 2. Subjects who received BIIB054 in Year 1 will continue with the same dose regimen in Year 2.

The total duration of study participation for each patient will be approximately 113 weeks, including a 5 -week screening period, a 48-week placebo-controlled treatment period, a 48-week active-treatment dose-blinded period, and a 12-week follow-up period.

\section{OUTCOME MEASURES:}

PRIMARY OUTCOME MEASURE: Percentage of participants with adverse events (AEs) and serious adverse events (SAEs) up to week 52.

\section{SECONDARY OUTCOME MEASURES:}

1. Change in striatal binding ratio (SBR) in putamen, striatum, and caudate (Timeframe: baseline, week

52). SBR will be measured by Striatal-Photon Emission Computed Tomography (SPECT) imaging of the Dopamine Transporter with Ioflupane ${ }^{123}$ I (DaTscan $\left.{ }^{\mathrm{TM}}\right)$.

2. Concentration of BIIB054 in the serum (Timeframe: baseline up to week 108).

3. Percentage of participants with anti-BIIB054 antibodies in the serum (Timeframe: baseline up to week 108).

\section{COMMENTS:}

As with all the other $\alpha$-synuclein targeting therapeutics, this is still an early phase study focusing on the safety and tolerability, however a number of the secondary and exploratory outcome measures will inform the design of the future studies. The study is enriched with a large number of biomarkers that hopefully will contribute to better mechanistic understanding of these targets.

\section{References}

1. https://www.sciencedirect.com/science/article/pii/S0969996118304480?via\%3Dihub.

2. http://n.neurology.org/content/90/15_Supplement/S26.001.

\section{LUNDBECK - Lu AF82422}

TITLE: Interventional, Randomized, Double-blind, Sequential-group, Placebo-controlled, Single-ascendingdose Study Investigating the Safety, Tolerability and Pharmacokinetic and Pharmacodynamic Properties of Lu AF82422 in Healthy Non-Japanese and Japanese Subjects and in Patients with Parkinson's Disease Phase I.

STATUS: Recruiting.

CLINICALTRIALS.GOV ID: NCT03611569.

SPONSOR: H. Lundbeck A/S.

ENROLMENT: 44.

ESTIMATED COMPLETION DATE: March 2020.

OBJECTIVE: To evaluate safety and tolerability, pharmacokinetics, and pharmacodynamics of Single Ascending Doses (SAD) of Lu AF82422 in Healthy Volunteers and Patients with Parkinson's Disease.

BACKGROUND: $\alpha$-synuclein targeting antibody.

STUDY DESIGN: Single ascending dose study. Cohort A: A1, A2, and A3: 24 healthy subjects in active arm and in placebo, with 8 subjects per cohort per treatment arm (aiming for an equal number of men and women). 
Cohort A4: 12 healthy subjects per treatment arm, with 6 non-Japanese subjects and 6 Japanese subjects (aiming for an equal number of men and women).

Part B: Cohort B1: 8 patients with PD in active arm: 8 in placebo.

\section{OUTCOME MEASURES:}

PRIMARY OUTCOME MEASURE: Adverse events.

SECONDARY OUTCOME MEASURES: spectrum of pharmacokinetic and pharmacodynamic measures including free/total $\alpha$-synuclein concentration in cerebrospinal fluid and plasma. Antidrug antibodies in serum will be measured as well.

\section{COMMENTS:}

This is an early phase SAD study that will define viability of the molecule to move into the more advanced stages of the development. No details on the profile of Lu AF82422 are provided.

\section{REFERENCES: No publications.}

\section{ROCHE/PROTHENA}

TITLE: A study to evaluate the efficacy of Prasinezumab (RO7046015/PRX002) in participants with early Parkinson's Disease (PASADENA) - Phase 2.

OBJECTIVE: Prasinezumab is a monoclonal antibody targeting $\alpha$-synuclein. The objective is to evaluate efficacy in early stage Parkinson's patients. Previously completed Phase I single and multiple ascending dose studies demonstrated good safety and tolerability of Prasinezumab, with robust binding of peripheral $\alpha$-synuclein and dose dependent increases of Prasinezumab in cerebrospinal fluid reaching concentrations that may be expected to engage aggregated $\alpha$-synuclein in the brain.

STATUS: On-going, recruitment completed.

CT.GOV ID: NCT03100149.

SPONSOR: Hoffmann-La Roche.

ENROLMENT: 300 patients. Inclusion criteria include a diagnosis of Parkinson's of less than two years, Modified Hoehn and Yahr score of 1 or 2, with no dopaminergic medication; and not expected to need it for the duration of the study. The only anti-Parkinson's medication allowed is MAO-B inhibitors.

ESTIMATED COMPLETION DATE: February 2021.

STUDY DESIGN: The PASADENA study has two parts, both of which are randomized, double-blind and placebo controlled. The first part has three arms:

ARM 1 - high dose Prasinezumab 4500mg for participants with body-weight greater than or equal to $(>/=) 65 \mathrm{~kg}$ or $3500 \mathrm{mg}$ for participants with body-weight less than $(<) 65 \mathrm{~kg}$.

ARM 2 - low dose Prasinezumab 1500mg.

ARM 3 - placebo.

All three arms will receive the relevant dose through IV infusion every 4 weeks for 52 weeks.

At the end of part 1, patients who have been on placebo will be randomized to either high dose or low dose Prasinezumab, joining those patients who have already been on the high or low dose. Part 2 will therefore have 2 arms: 
HIGH DOSE ARM - high dose group participants and placebo group participants randomized to high dose level will receive Prasinezumab at high dose level as intravenous infusion every 4 weeks for additional 52 weeks.

LOW DOSE ARM - low dose group participants and placebo group participants randomized to low dose level will receive Prasinezumab at low dose level as intravenous infusion every 4 weeks for additional 52 weeks.

\section{OUTCOME MEASURES:}

Primary Outcome Measures:

1. Change From Baseline in Movement Disorder Society-Unified Parkinson's Disease Rating Scale (MDSUPDRS) Total Score (Sum of Parts I, II, and III) at Week 52 [Time Frame: Baseline and Week 52].

\section{Secondary Outcome Measures:}

1. Change From Baseline in Dopamine Transporter Imaging With Single Photon Emission Computed Tomography (DaT-SPECT) Signal at Week 52 [Time Frame: Baseline and Week 52].

2. Change From Baseline in the MDS-UPDRS Motor Subscale (Part III) Score [Time Frame: Baseline and Week 52].

3. Clinical Global Impression of Improvement (CGI-I) Score at Weeks 24 and 52 [Time Frame: Week 24 and Week 52].

4. Patient Global Impression of Change (PGIC) Score at Weeks 24 and 52 [Time Frame: Week 24 and Week 52].

5. Time to Start of Dopaminergic Symptomatic Treatment [Time Frame: From Baseline to Week 52].

6. Percentage of Participants With Adverse Events (AEs) and Serious AEs (SAEs) [Time Frame: From Day 1 to Week 104].

7. Percentage of Participants With Anti-Drug Antibodies (ADAs) Against R07046015 [ Time Frame: Baseline, Pre-dose (0 hours) on Weeks 4, 20, 36, 52, 56, 68, 80, and 104; at early termination (up to Week 104), and follow-up (12 weeks after last dose up to Week 116)].

8. Systemic Clearance (CL) of RO7046015 [ Time Frame: Predose (0 hours) and end of infusion (infusion length $=2$ hours or less) on Day 1, Weeks 4, 20,36, 52, 56, 68, 80, and 104; at Day 7, Day 14, early termination (up to Week 104), and follow-up (12 weeks after last dose up to Week 116)].

9. Apparent Volume of Distribution $(\mathrm{Vz} / \mathrm{F})$ of RO7046015 [Time Frame: Predose ( 0 hours) and end of infusion (infusion length $=2$ hours or less) on Day 1 , Weeks 4, 20,36, 52, 56, 68, 80, and 104; at Day 7, Day 14, early termination (up to Week 104), and follow-up (12 weeks after last dose up to Week 116)].

10. Area Under the Serum Concentration-Time Curve (AUC) of RO7046015 [Time Frame: Predose (0 hours) and end of infusion (infusion length $=2$ hours or less) on Day 1, Weeks 4, 20, 36, 52, 56, 68, 80, and 104; at Day 7, Day 14, early termination (up to Week 104), and follow-up (12 weeks after last dose up to Week 116)].

11. Maximum Observed Serum Concentration (Cmax) of RO7046015 [Time Frame: Predose (0 hours) and end of infusion (infusion length $=2$ hours or less) on Day 1, Weeks 4, 20, 36, 52, 56, 68, 80, and 104; at Day 7, Day 14, early termination (up to Week 104), and follow-up (12 weeks after last dose up to Week 116)].

12. Minimum Observed Serum Trough Concentration (Ctrough) of RO7046015 [Time Frame: Predose $(0$ hours) and end of infusion (infusion length $=2$ hours or less) on Day 1, Weeks 4, 20, 36, 52, 56, 68, 80, and 104; at Day 7, Day 14, early termination (up to Week 104), and follow-up (12 weeks after last dose up to Week 116)].

\section{COMMENTS:}

This is a comprehensive phase 2 study assessing both efficacy and safety/tolerability at two active dose levels of Prasinezumab. 


\section{OTHER $\alpha$-SYNUCLEIN TARGETING APPROACHES UNIVERSITY OF COLORADO - GLYCEROL PHENYLBUTYRATE}

TITLE: Phenylbutyrate Response as a Biomarker for Alpha-synuclein Clearance from the Brain. Open Label Phase I.

STATUS: Active, NOT recruiting.

CLINICALTRIALS.GOV ID: NCT02046434.

SPONSOR: University of Colorado, Denver.

ENROLMENT: 40.

ESTIMATED COMPLETION DATE: Fall 2018.

OBJECTIVE: This is a Phase I clinical trial of the FDA approved drug Glycerol Phenylbutyrate to see if phenylbutyrate can increase the removal of $\alpha$-synuclein from the brain into the bloodstream.

BACKGROUND: Reduction of $\alpha$-synuclein aggregation and increase in clearance.

STUDY DESIGN: This is a Phase I clinical trial of phenylbutyrate in 20 Parkinson patients and 20 age- and sex-matched normal control subjects to see if phenylbutyrate can increase the removal of alpha-synuclein from the brain into the bloodstream. All subjects will receive 20 grams/day of phenylbutyrate in the liquid form phenylbutyrate-triglyceride taken as one teaspoonful three times per day with meals. Blood will be drawn on two days prior to starting phenylbutyrate to measure alpha-synuclein concentrations. Phenylbutyrate-triglyceride will then be started and the change in plasma alpha-synuclein will be measured on day 1, 7, 14, and 21 days while taking phenylbutyrate. After 21 days, the drug will be stopped and a final blood sample will be measured at 28 days to see if plasma alpha-synuclein has fallen to its pre-phenylbutyrate level. No effects on Parkinson symptoms are expected during this short trial.

\section{OUTCOME MEASURES:}

PRIMARY OUTCOME MEASURE: Levels of $\alpha$-synuclein in blood plasma.

SECONDARY OUTCOME MEASURES: Not listed.

\section{COMMENTS:}

This is an open label proof of principle study. The study has been completed but the results have not been published. The team is pursuing a Phase II dose selection study.

REFERENCES: No publications.

\section{NEUROPORE THERAPIES/UCB - NPT200-11}

TITLE: A Phase 1, Randomized, Double-Blind, Single Ascending Dose Trial of the Safety, Tolerability and Pharmacokinetics of NPT200-11 in Healthy Subjects.

STATUS: Completed.

CLINICALTRIALS.GOV ID: NCT02606682.

SPONSOR: Neuropore Therapies/UCB.

ENROLMENT: 55 .

COMPLETION DATE: February 2016. 
OBJECTIVE: To evaluate safety and tolerability, pharmacokinetics, and pharmacodynamics of Single Ascending Doses (SAD) of NPT200-11 in Healthy Volunteers and Patients with Parkinson's Disease.

BACKGROUND: NPT200-11 is a small molecule inhibitor of $\alpha$-synuclein dimerization, preventing further propagation to oligomers and fibrils.

STUDY DESIGN: Single ascending dose of orally administered capsule(s) NPT200-11 vs placebo: 15, 30, $60,120,240,360$ and $480 \mathrm{mg}$ dose.

\section{OUTCOME MEASURES:}

PRIMARY OUTCOME MEASURE: Safety, including adverse events, physical examinations, ECGs, clinical laboratory tests. Time Frame: Screening (28 days prior to dosing) through Day 7.

SECONDARY OUTCOME MEASURES: To possibly determine the maximally tolerated dose (MTD) of orally administered NPT200-11 in healthy subjects. The number of participants with unacceptable toxicities at each dose level will determine the maximally tolerated dose.

\section{COMMENTS:}

An early phase SAD study was completed but the data have not been published and no information on future drug development are available.

\section{REFERENCES: No publications.}

\section{PD-NILOTINIB AND NILO-PD - NILOTINIB}

BACKGROUND: Nilotinib, a c-ABL inhibitor, promotes autophagic degradation of alpha-Synuclein and p-Tau and was shown to be neuroprotective in animal models of parkinsonism. A small open label clinical study tested the safety/tolerability of nilotinib in PD for the first time and reported that there were positive, though exploratory, signs of efficacy [1].

\section{PD NILOTINIB}

Impact of Nilotinib on Safety, Tolerability, Pharmacokinetics and Biomarkers in Parkinson's Disease (PD Nilotinib).

STATUS: Recruitment completed.

CLINICALTRIALS.GOV ID: NCT02954978.

SPONSOR: Georgetown University.

ENROLMENT: 75.

EXPECTED COMPLETION DATE: July 2020.

\section{NILO-PD}

Nilotinib in Parkinson's Disease (NILO-PD).

STATUS: On-going, recruitment completed.

CLINICALTRIALS.GOV ID: NCT03205488.

SPONSOR: Northwestern University / Michael J Fox Foundation (MJFF).

ENROLMENT: 75.

EXPECTED COMPLETION DATE: October 2020. 
OBJECTIVE: To evaluate safety and tolerability of daily oral administration of nilotinib (150-300mg once daily) in Parkinson's Disease.

STUDY DESIGN: both studies have similar design. Out of 75 participants that will be recruited and randomly assigned 1:1:1 to the 3 groups (arms), 150mg: 300mg: placebo; all once daily. Intervention duration: 6 months and 2 months follow up (NCT03205488) and 12 months and 3 months follow up (NCT02954978).

\section{OUTCOME MEASURES:}

PRIMARY OUTCOME MEASURE: Safety and tolerability (both studies).

\section{SECONDARY OUTCOME MEASURES:}

Unified Parkinson's Disease Rating Scale (MDS-UPDRS) part III (motor function) will be evaluated to determine if Nilotinib has the potential to reduce the MDS-UPDRS motor score as well as a spectrum of motor and non-motor outcome scales, PK profile in cerebrospinal fluid and a number of exploratory biomarkers.

\section{COMMENTS:}

Nilotinib is a drug approved for treatment of chronic leukaemia and as such has a number of safety concerns. The current studies aim to assess safety/ tolerability of nilotinib as well as explore signals of efficacy to determine if the future studies are warranted. There are other molecules targeting the c-ABL pathway in development with potentially better safety profile and better CNS penetration, (Sun Pharma, NCT02970019).

\section{References}

1. Pagan, F., et al., Nilotinib Effects in Parkinson's disease and Dementia with Lewy bodies. J Parkinsons Dis, 2016.

\section{RESOURCES}

\section{PARKINSON'S THERAPIES IN DEVELOPMENT}

The Hope List - https://drive.google.com/file/d/1nf-r71qaoVFUDERwk6cNG3zG_YqSI1pI/view?usp=sharing.

\section{FINDING A CLINICAL TRIAL}

ClinicalTrials.gov from the US National Library of Medicine - https://clinicaltrials.gov.

PD Trial Tracker; analyzing ClinicalTrials.gov for Parkinson's specific trials - http://www.pdtrialtracker.info.

Fox Trial Finder - https://foxtrialfinder.michaeljfox.org.

European Parkinson's Disease Association - https://www.epda.eu.com/about-parkinsons/treatments/clinicaltrials/.

Parkinson's UK - https://www.parkinsons.org.uk/research/take-part-research.

UK NHS Clinical Trials Gateway - https://www.ukctg.nihr.ac.uk.

Cure Parkinson's Trust - https://www.parkinsonsmovement.com/clinical-trials/.

Parkinson's Study Group - http://www.parkinson-study-group.org/clinical-trials.

American Parkinson Disease Association - https://www.apdaparkinson.org/resources-support/living-withparkinsons-disease/clinical-trials/.

CenterWatch - https://www.centerwatch.com/clinical-trials/listings/condition/117/parkinsons-disease/.

\section{WHAT DOES IT MEAN TO PARTICIPATE IN A PARKINSON'S CLINICAL TRIAL?}

Michael J Fox Foundation, Clinical Trial Companion - https://www.michaeljfox.org/pdcompanion.html Parkinson's Foundation - https://www.parkinson.org/Understanding-Parkinsons/Treatment/Clinical-Trials. 